\title{
Video Transmission over MIMO-OFDM System: MDC and Space-Time Coding-Based Approaches
}

\author{
Haifeng Zheng, ${ }^{1}$ Congchong $\mathrm{Ru}^{2}{ }^{2}$ Chang Wen Chen, ${ }^{3}$ and Lun $\mathrm{Yu}^{1}$ \\ ${ }^{1}$ Department of Information and Communication Engineering, Fuzhou University, Fuzhou, Fujian 350002, China \\ ${ }^{2}$ Department of Electronic Engineering, Tsinghua University, Beijing 100084, China \\ ${ }^{3}$ Department of Electrical and Computer Engineering, Florida Institute of Technology, Melbourne, FL 32940, USA
}

Received 16 November 2006; Accepted 21 December 2006

Recommended by Jianfei Cai

MIMO-OFDM is a promising technique for the broadband wireless communication system. In this paper, we propose a novel scheme that integrates multiple-description coding (MDC), error-resilient video coding, and unequal error protection strategy with hybrid space-time coding structure for robust video transmission over MIMO-OFDM system. The proposed MDC coder generates multiple bitstreams of equal importance which are very suitable for multiple-antennas system. Furthermore, according to the contribution to the reconstructed video quality, we apply unequal error protection strategy using BLAST and STBC space-time codes for each video bitstream. Experimental results have demonstrated that the proposed scheme can be an excellent alternative to achieve desired tradeoff between the reconstructed video quality and the transmission efficiency.

Copyright (c) 2007 Haifeng Zheng et al. This is an open access article distributed under the Creative Commons Attribution License, which permits unrestricted use, distribution, and reproduction in any medium, provided the original work is properly cited.

\section{INTRODUCTION}

Video communication over wireless network has been a significant challenge for current multimedia technology. As it is well known, wireless channels often suffer from multipath fading, shadowing, intersymbol interference, and so forth. Meanwhile, compressed video is very sensitive to error-prone environment. Any transmission error may lead to the loss of decoding synchronization and severe degradation to the received video quality.

Fortunately, great progresses have been made in the recent development of wireless communication and video transmission. Orthogonal frequency-division multiplexing (OFDM) has become a promising technique for transmission of signals in the broadband wireless communication systems. Moreover, multiple antennas system with multiple transmitters and multiple receivers, called a multiple-input and multiple-output (MIMO) system, has been shown to be an effective way to transmit high data rate over wireless channels. Therefore, OFDM in conjunction with multiple-input and multiple-output is not only able to enhance the capacity of system but also able to combat the channel fading and interference effectively.

It is widely believed that multiple-transmit and-receive antennas can improve the performance of wireless systems.
Actually, this benefit can be exploited in two ways: spatial diversity and spatial multiplexing. Spatial diversity can improve the reliability of reception to combat channel fading by sending signals that carry the same information through independent paths. Such diversity schemes can be implemented using trellis-based space-time codes and orthogonal designs. For example, space-time block codes (STBCs) have been proposed to achieve diversity gains [1]. On the other hand, spatial multiplexing can provide higher data rate by transmitting different data streams in parallel through independent channels. Several schemes have been proposed to exploit the benefit of spatial multiplexing phenomenon. For example, Bell Labs space-time architecture (BLAST) [2] is such a scheme. In another words, multiple-transmit and-receive antennas can be used to provide higher reliability of reception using spatial diversity or give higher throughout using spatial multiplexing. In this paper, we will exploit the benefits from both types of these two schemes for robust video transmission over wireless channels.

Meanwhile, in image and video transmission applications, several approaches have been proposed to improve the robustness of image and video transmission over error-prone network such as Internet and wireless network. Multipledescription coding (MDC) is such an effective source coding method. MDC generates multiple encoded bitstreams 

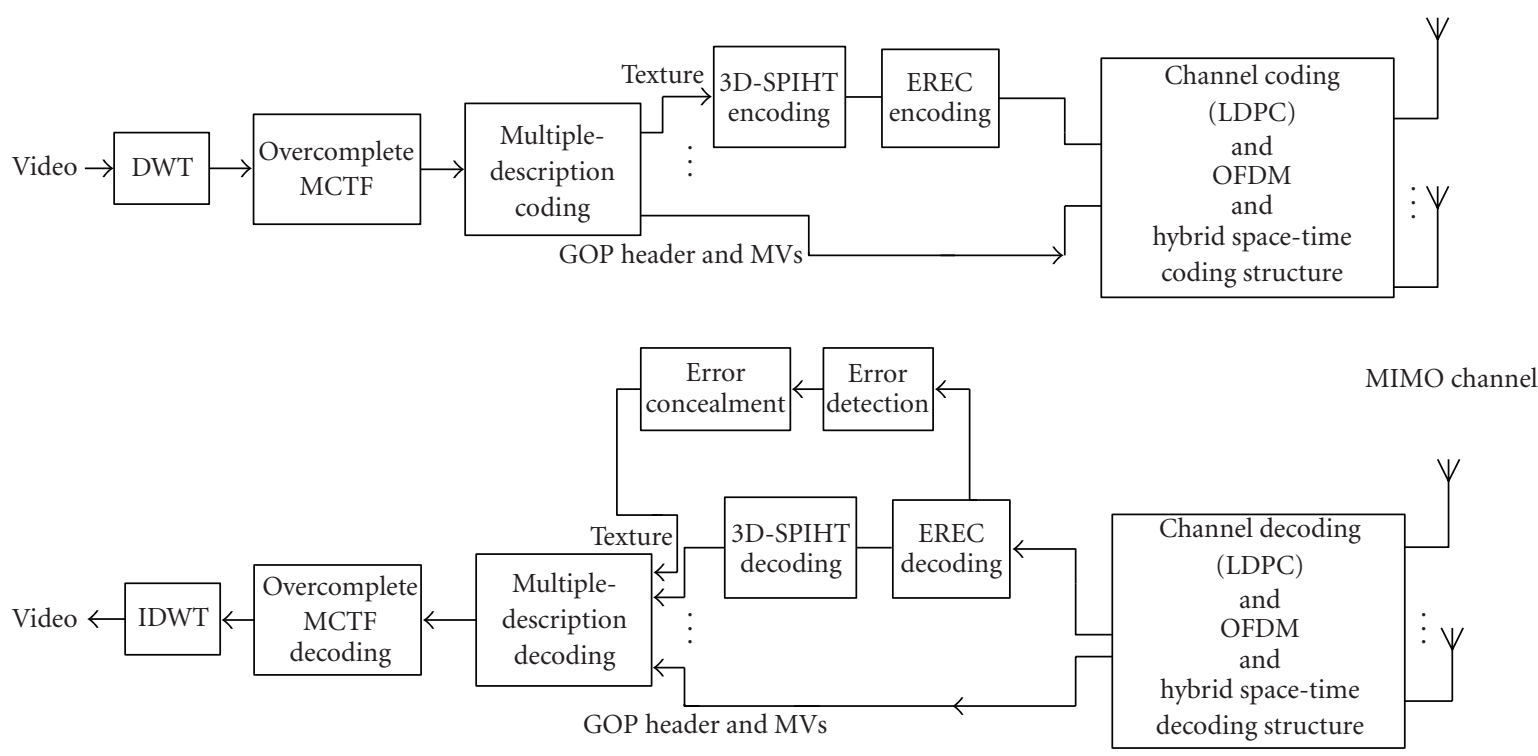

FIGURE 1: The overall block diagram of the proposed system.

that are equally important and independent. The objective of MDC is that if all bitstreams have been received correctly, a high signal quality can be reconstructed, whereas, if some bitstreams have been lost, a low-quality, but acceptable signal quality can still be reconstructed from the received description. MDC uses the idea of diversity to transmit bitstreams along different channels with the premise that each channel experiences independent failure events, such that the probability of receiving the information from each channel is equal. This assumption is also true in MIMO channel case. In a system with transmit and receive antennas, the channel between individual antenna pairs is assumed to be independent and identically distributed (i.i.d.). Moreover, the probability of all channels between antenna pairs falling into deep fading simultaneously is small. Therefore, MDC is also very suitable for robust video transmission over multiple antennas system

There have been several works to report video transmission over multiple antennas system [3-7]. However, none of these existing schemes have explored the integration of data bitstreams transmission using MDC and MIMO-OFDM. In this research, we construct a new system that integrates multiple-description coding, error-resilient video coding, unequal error protection scheme using hybrid space-time coding structure for robust video transmission over MIMO-OFDM system. In this paper, we propose a multiple-description coding scheme based on wavelet video coding, where each generated description still retains the main quality of the original image. In the present of lost descriptions, we can perform error concealment method to compensate the lost descriptions without introducing some amount of redundancy between the descriptions. Unlike traditional multiple-description schemes, the proposed multiple-description algorithm enables us to generate more than two bitstreams that may be more appropriate for multiple-antenna transmission of compressed video.
In addition, to improve the error resilience of each description, each description of the compressed video will be further decomposed into multiple bitstreams. We also introduced error-resilient entropy coding (EREC) [8] to solve the problems of packetization and synchronization for multiple bitstreams transmission. Furthermore, as mentioned before, in MIMO system, spatial diversity performs more robustly, while spatial multiplexing provides higher data rate increment when given a fixed reliability level. This work is inspired by this idea. In this work, diversity scheme can be used to achieve a better error protection for important data such as motion vectors and GOP header information, whereas multiplexing scheme can be applied to obtain high transmission data rate. Therefore, we use such hybrid space-time coding structure to achieve unequal error protection for video transmission.

The rest of the paper is organized as follows. The proposed transmission scheme is described in Section 2. Then, simulation results are presented in Section 3 to demonstrate the effectiveness of the proposed scheme. Finally, we conclude in Section 4 with some discussion.

\section{THE PROPOSED SCHEME}

Figure 1 shows the overall block diagram of the proposed system. At the encoder side, the input video sequence is first divided into groups of pictures (GOPs). And then, within a GOP, each frame is hierarchically decomposed by the critically sampled discrete wavelet transform (DWT). Furthermore, we adopt the motion-compensation-based approach to decorrelate the video signal along the temporal dimension, which jointly implements the temporal WT and MC. To avoid the shift-variant problem existing inherently in critically sampled discrete wavelet transform and to achieve high coding performance, motion estimation and 
motion compensation are performed in the overcomplete wavelet domain, which is so called the overcomplete motioncompensated temporal filtering (OMCTF). After the overcomplete motion-compensated temporal filtering operation, the generated texture information and motion vector information are distributed into each description by a multipledescription algorithm. In this work, four descriptions are generated from the encoded bitstreams. For the coding of residual wavelet coefficients in each description, we develop a modified 3D-SPIHT algorithm [9], which is not a blockbased coding algorithm and is suitable for our MDC approach. As discussed before, in order to make each description still resilient to channel errors, we partition wavelet coefficients into a number of independent spatial orientation trees, which is borrowed from Creusere's work [10], and each spatial orientation tree can be encoded and decoded independently. Thus, an error in the bitstream belonging to one tree does not affect the others. However, such an algorithm is still sensitive to bit errors, because each spatial orientation tree produces variable-length bitstreams. A single bit error may lead to loss of synchronization for each spatial orientation tree. In order to generate entropy-coded video bitstreams that are self-synchronized for robust decoding at the receiving end, we also introduce EREC to generate fiexed-length bitstreams, and then each description is encoded by channel coding. In this scheme, we use low-density parity-check codes (LDPCs). After channel coding, each antenna employs an OFDM modulator with $N$ subcarriers. As mentioned above, hybrid space-time coding structure using STBC combined with BLAST is then applied according to different priority levels of the bitstreams. In this scheme, we allocate different numbers of the subcarriers to different space-time coders according to the proportion between important bits and less important bits. In the decoding stage, the signal at the receiver can be separated and recovered from different subcarriers. In the following sections, we will discuss more technical details for this scheme.

\subsection{Multiple-description and error-resilient video coding}

\subsubsection{Multiple-description coding based on OMCTF}

Recently, motion-compensated temporal filtering (MCTF) has been proposed to replaced motion-compensated prediction (MCP) for developing scalable video coder. Compared to MCP, MCTF is an open-loop structure, which does not employ recursive structure for ME/MC. Motioncompensated temporal filtering is firstly proposed by $\mathrm{Ohm}$ and improved by Choi and Woods. Motion-compensated temporal filtering is able to not only remove the redundancy between interframes using motion estimation and motion compensation, but it also removes the temporal redundancy by a pyramidal temporal decomposition structure. MCTF has two forms, which are denoted as spatial-domain MCTF and overcomplete MCTF (OMCTF) or in-band MCTF [11]. The difference between them is that MCTF is performed in the original frames in spatial-domain MCTF, otherwise,
MCTF is performed in the wavelet domain in overcomplete MCTF. However, in the architecture of overcomplete MCTF, due to the problem caused by shift-variant property, important information of motion accuracy would be lost if motion estimation and motion compensation (ME/MC) are performed only in critically sampled wavelet domain. Therefore, to achieve high coding efficiency in the overcomplete MCTF, ME/MC is performed in overcomplete wavelet domain. Moreover, overcomplete motion-compensated temporal filtering provides flexible scalable feature for scalable video coding. Our MDC scheme is based on overcomplete MCTF wavelet video coding.

Many MD approaches introduce the correlation between descriptions by inserting some amount of redundancy.However, a class of MD approaches assumes that there is a strong correlation between adjacent coefficients so that the value of given coefficients can be reasonably predicted by the value of their neighbors. Several such MD schemes have been proposed for image and video applications [12-14]. Tanabe and Farvardin have shown that wavelet coefficients in the lowest frequency subband have similar spatial correlation with that of the original image [15]. This means that this characteristic can be utilized to construct an MD wavelet-based video coder. By exploiting the nature correlation of the adjacent coefficients in the wavelet domain, it is possible to develop a multiple-description algorithm under the framework of overcomplete motion-compensated temporal filtering. As mentioned before, overcomplete motion-compensated temporal filtering jointly implements the spatial wavelet transform (WT) and ME/MC in the temporal direction to decorrelate the video signal. Thus, the frames within a GOP after performing overcomplete motion-compensated temporal filtering, the correlation of the signal will be further decorrelated except the lowest frequency subband in the temporal lowest frequency frame. Here we denote the temporal lowest frequency frame as LLL frame. Therefore, what should be carefully considered is how to make use of the correlation of the coefficients in the lowest subband frequency in the LLL frame. In order to exploit this correlation, adjacent wavelet coefficients should be dispersively distributed into different descriptions. An example for this method is shown in Figure 2. As depicted in Figure 2, different circles represent wavelet coefficients in different descriptions. In the example for four descriptions, the wavelet coefficients in the lowest frequency subband in the LLL frame are equally divided into different descriptions. The other wavelet coefficients in the high subband in the LLL frame will be partitioned into four descriptions as shown in Figure 2. The partitioning of the other wavelet coefficients in the other frames within a GOP will be the same as that of LLL frame.

Another key problem in such an MD scheme is how to partition the wavelet coefficients into different descriptions. As it is known, there is a direct relationship between wavelet coefficients in the different subbands, which is called parentchild orientation tree structure. Several wavelet-based image coding algorithms based on this idea have been developed, which have demonstrated excellent coding efficiency $[16,17]$. In this scheme, we use the 3D-EZW tree structure 


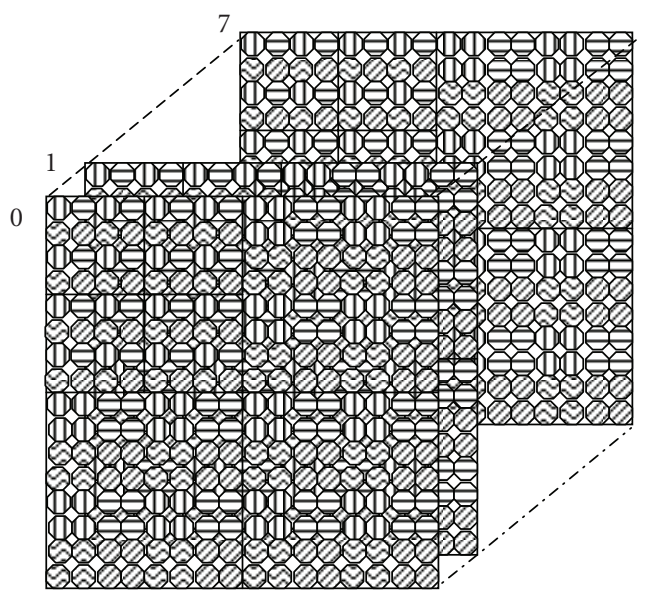

Figure 2: An example of partitioning strategy.

[18]. Figure 2 also illustrates the tree structure. Meanwhile, in order to improve the performance of error resilience, we should further divide each description into several packets. In this scheme, we packetize each orientation tree into one packet. For robustness, we also expect that each packet could be encoded and decoded independently. Thus, the decoding failure in one packet will not affect the others. This is a welcome feature for robust image and video transmission over error-prone environment.

\subsubsection{EREC and error concealment}

When compressed video is transmitted over wireless channel, a single bit error may cause the error to propagate not only to frames along the temporal direction, but also within an individual frame. The problem that causes such error propagation is that VLC coding scheme is usually adopted for coding the texture information after ME/MC. As mentioned before, each orientation tree is encoded and decoded independently. To balance the quality for each orientation tree, we encode each orientation tree to the same bitplane. A single bit error may cause the loss of synchronization among these orientation trees. To overcome this drawback, we need to design a scheme that is able to limit the bit error within each orientation tree.

Error-resilient entropy coding (EREC) is an effective means to recover lost or erroneous information to enhance error resilience for coding variable-length blocks of data. The scheme is originally proposed by Redmill and Kingsbury to handle the sequential transmission of DCT coded data blocks over noisy channels. The key of the EREC is to reorganize the variable-length data blocks into fixed-length slots with negligibly increased data size. Figure 3 shows EREC bit reorganization algorithm. It has been shown that the EREC can significantly reduce the channel error propagation effects and that remaining channel error propagation is most likely to affect data from the end of longer blocks [8]. The characteristic of EREC decoding is very suitable for some embedded coding methods, such as 3D-SPIHT algorithm. As we

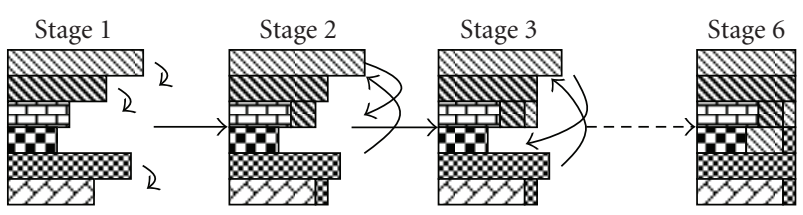

FIGURE 3: EREC bit reorganization algorithm.

know, 3D-SPIHT encodes the wavelet coefficients from high bitplane to low bit-plane by threshold. This suggests that the encoded bitstream contains more important information in the beginning of the bitstream. Therefore, to further enhance the error resilience of the proposed scheme, we apply EREC to the symbols coded by 3D-SPIHT algorithm to avoid synchronization loss among the orientation trees.

As we know, EREC decoding needs a priori known information about the position where it should stop. There are usually three methods to attain this purpose: adding special bitstream end symbols, coding a length before each bittream, or coding bitstream to a known bitplane. The first two methods will reduce the coding efficiency due to adding a certain overhead. In this work, we adopt the third approach, that is, encoding each orientation tree to the same bitplane. Thus, each slot will have an explicit ending position. This method also keeps the balanced quality for each orientation tree because each orientation tree stops decoding at the same threshold value. Another key problem in the EREC algorithm is how to detect error during EREC decoding. In [19], Cao and Chen proposed a method for image wavelet-based coding. They added one bit for the first several bits in each slot data for the purpose of error detection in the EREC encoding stage. This is because the first several bits coded by SPIHT have higher energy than other bits. However, this scheme does not consider that each wavelet tree may not have the same energy distribution. Therefore, if one bit is added at the fixed position in the first several bits for each wavelet tree, some slots with errors that still contain high energy may miss the error detection. In this work, we add one bit for parity check at the tail of each bitplane for the first three bitplanes, respectively. Thus, if we detect any error in these parity-check bits in the slot, we will regard the slot corrupted. If some slots are detected with errors, they will not take part in the decoding stage.

Meanwhile, additional advantage of the proposed scheme using wavelet tree coding combined with EREC in MD scheme is that simple error concealment methods can be employed to estimate the lost information. Since MDC strategy assumes that not all bitstreams in the different descriptions experience failures simultaneously, thus, if we detect the errors occurring in one slot in some description, most likely we can apply error concealment method to estimate the lost information from the neighboring uncorrupted slots in the other descriptions. As mentioned before, the advantage of the MDC algorithm proposed in this scheme is that there exists strong correlation between the adjacent wavelet coefficients in the lowest frequency subband in the LLL frame. Therefore, the lost wavelet coefficients can be estimated by 
calculating the average of their neighboring coefficients by decoding the corresponding slots. In this scheme, error concealment is only carried out in the lowest subband in the LLL frame.

\subsection{Unequal error protection using BLAST and STBC}

In this paper, we consider a MIMO-OFDM system with $M_{t}\left(M_{t}=4\right)$ transmit and $M_{r}\left(M_{r}=4\right)$ receiver antennas for robust video transmission. Each antenna employs an OFDM modulator with $N$ subcarriers. Transmitting signals of different subcarriers will be transmitted simultaneously over all transmit antennas. As mentioned before, the coded source bits transmitted over each antenna also contain unequally important information according to their contribution to the decoding process. Thus, data partitioning can be used in this scheme. As we know, data partition is a very effective tool for the transmission of video over an error environment. Data partitioning divides a coded signal bitstream into two components. In this scheme, each description is further divided into two partitions: motion vectors and GOP header information, and texture information. Due to their different priority levels, we can carry out unequal error protection strategy. Motivated by this, we propose a hybrid space-time coding structure to transmit multiple bitstreams generated by multiple-description coding scheme as discussed in the previous section. In this research, we combine BLAST and STBC in this multiple antennas system to obtain unequal error protection for robust video transmission. By transmitting the most critical information using MIMO diversity scheme, the average reconstructed quality at the receiver will not degrade largely. Meanwhile, by sending the texture information at each antenna simultaneously using MIMO multiplexing scheme, the transmission data rate will be enhanced. The illustration of the proposed UEP scheme is shown in Figure 4.

As we have discussed, the coded source bits might be divided into two parts according to their contribution to the decoding process, namely TINF, which stands for the texture information, and MIB, which presents the more important bits including motion vectors and GOP header information. Considering the independent channels generated by BLAST, it is intuitively suitable for the transmission of TINF bits. Additionally, the transmission efficiency will also be increased greatly. However, MIB is a crucial component in a video codec. For example, even one bit error in the MIB bits may cause a decoding collapse or cause serious error propagation within a GOP and greatly degraded video quality. Compared with BLAST, STBC achieves full diversity gain. It performs much better than BLAST in terms of BER, however, with much lower transmission efficiency. Consequently, to guarantee tradeoff between reconstructed video quality and transmission efficiency, we apply different space-time coding methods in the scheme, as shown in Figure 4. In this scheme, we employ four transmit antennas to match the four MDC bitstreams from the video encoder. Assuming the space-time code rate is $R$, MIB has $B_{\mathrm{MIB}}$ bits and TINF has $B_{\text {TINF }}$ bits, we will have $P=\left\lfloor B_{\mathrm{MIB}} N /\left(B_{\mathrm{TINF}} R+B_{\mathrm{MIB}}\right)\right\rfloor$ subcarriers to transmit MIB. These subcarriers are denoted as $\left\{\begin{array}{llll}f_{1} & f_{2} & \cdots & f_{P}\end{array}\right\}$.

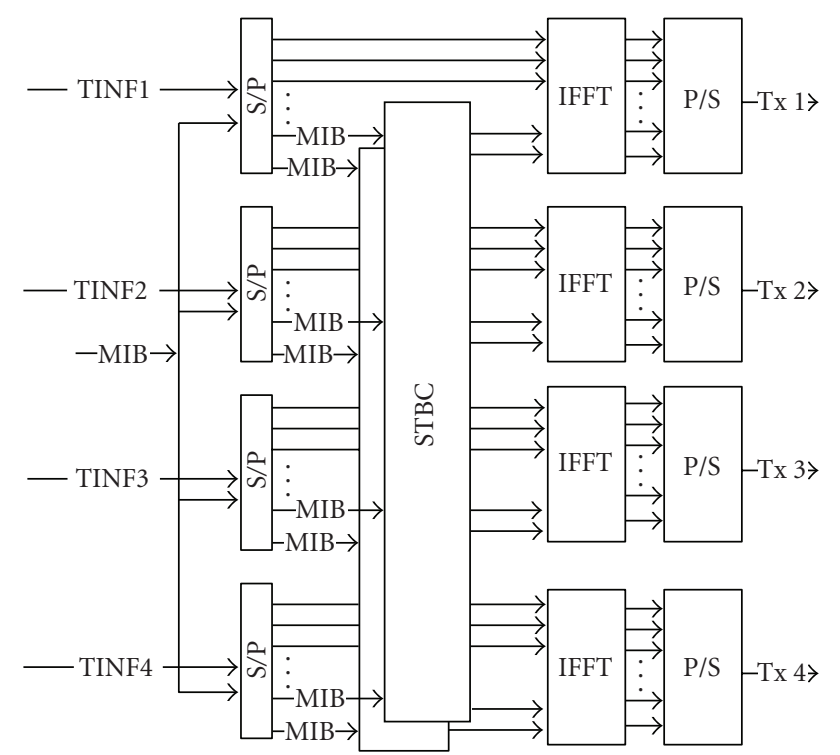

FIGURE 4: The block diagram of the proposed UEP scheme.

\subsubsection{BLAST}

V-BLAST structure is employed in this scheme. At the transmitter, each MDC stream is assigned to one antenna which holds an independent transmit channel as mentioned before. At the receiver, the frequency-domain signals at subcarrier $\left\{\begin{array}{llll}f_{P+1} & f_{P+2} & \cdots & f_{N}\end{array}\right\}$ will be sent to a simple ZF detector [10] to recover the transmit symbols. Note that the optimal detect algorithms might bring better performance, but the process complexity and delay would be increased together. An efficient way to solve this problem is to import channel codes. In this scheme, the LDPC code is employed [20]. As a result, the transmission performance can be greatly increased.

\subsubsection{STBC}

For four-transmit-antenna system, assuming that the channel is stable during four OFDM symbol periods, we construct a 3/4 rate STBC code:

C

$$
=\left(\begin{array}{cccc}
c_{1} & -c_{2}^{*} & \frac{1}{\sqrt{2}} c_{3}^{*} & \frac{1}{\sqrt{2}} c_{3}^{*} \\
c_{2} & c_{1}^{*} & \frac{1}{\sqrt{2}} c_{3}^{*} & -\frac{1}{\sqrt{2}} c_{3}^{*} \\
\frac{1}{\sqrt{2}} c_{3} & \frac{1}{\sqrt{2}} c_{3} & -\frac{1}{2}\left(c_{1}+c_{1}^{*}-c_{2}+c_{2}^{*}\right) & \frac{1}{2}\left(c_{1}-c_{1}^{*}+c_{2}+c_{2}^{*}\right) \\
\frac{1}{\sqrt{2}} c_{3} & \frac{1}{\sqrt{2}} c_{3} & \frac{1}{2}\left(c_{1}-c_{1}^{*}-c_{2}+c_{2}^{*}\right) & -\frac{1}{2}\left(c_{1}+c_{1}^{*}+c_{2}-c_{2}^{*}\right)
\end{array}\right) .
$$

It is obvious that three complex symbols per subcarrier are transmitted during four OFDM symbol periods. At the receiver, in order to obtain an orthogonal channel matrix, we define the received signal at the $j$ th receiving antenna, $n$th 
subcarrier as

$$
\begin{aligned}
Y_{j}(n)= & {\left[\begin{array}{llll}
y_{j}^{1}(n) & y_{j}^{2}(n) & y_{j}^{3}(n) & y_{j}^{4}(n)
\end{array}\right.} \\
& \left(y_{j}^{1}(n)\right)^{*}\left(\begin{array}{llll}
\left.y_{j}^{2}(n)\right)^{*} & \left(y_{j}^{3}(n)\right)^{*} & \left(y_{j}^{4}(n)\right)^{*}
\end{array}\right] .
\end{aligned}
$$

Here $(\bullet)^{*}$ denotes conjugate operation and $y_{j}^{t}(n)$ presents the received signal at the $t$ th OFDM symbol period.

Then the transmit symbol can be recovered as

$$
\widehat{c}(n)=\frac{1}{M_{r} * \sum_{i=1}^{M_{t}}\left\|h_{i, j}\right\|^{2}} \sum_{j=1}^{M_{r}} H_{j}^{H}(n) * Y_{j}(n),
$$

where $\hat{c}(n)=\left[\begin{array}{llllll}\hat{c}_{1}^{*}(n) & \hat{c}_{2}^{*}(n) & \hat{c}_{3}^{*}(n) & \hat{c}_{1}(n) & \hat{c}_{2}(n) & \hat{c}_{3}(n)\end{array}\right]^{T}$, and $H_{j}(n)$ is the orthogonal channel matrix at the subcarrier $n$ in the receive antenna $j$, and $(\bullet)^{H}$ denotes the conjugate transpose operation. Finally, the video-coded bit sequence will be ready for decoding after constellation demapping and bits rearrangement.

\subsection{Channel model}

We consider a frequency-selective Rayleigh fading channel which has $L$ independent delay paths with arbitrary delay power profiles. The baseband equivalent channel can be modeled as

$$
h_{i, j}^{k}=\sum_{l=0}^{L-1} \alpha_{i, j}^{k}(l) \sigma\left(t-\tau_{l}\right)
$$

where $\alpha_{i, j}^{k}(l)$ is the path gain coefficient of the $l$ th path between transmit antenna $i$ and receiver antenna $j$ at the $k$ th OFDM symbol period, and $\tau_{l}$ presents the $l$ th path delay. The $\alpha_{i, j}^{k}(l)$ is modeled as zero-mean complex Gaussian random variable with variance $E\left|\alpha_{i, j}^{k}(l)\right|^{2}=\sigma_{l}^{2}$. The channel coefficients are assumed spatially uncorrelated.

The received signal at $n$th subcarrier at $j$ th receive antenna during $k$ th OFDM block may be denoted as

$$
y_{j}^{k}(n)=\sqrt{\rho} \sum_{i=1}^{M_{t}} x_{i}^{k}(n) H_{i, j}^{k}(n)+\omega_{j}^{k}(n),
$$

where $\rho$ is the average signal-to-noise ratio per receiver and $H_{i, j}^{k}(n)=\sum_{l=0}^{L-1} \alpha_{i, j}^{k}(l) e^{-j 2 \pi n \Delta f \tau_{l}}$ is the subchannel gain. Here $\Delta f=1 / T_{s}$ is the inter-subchannel space and $T_{s}$ is the OFDM symbol period. The additive noise $\omega_{j}^{k}(n)$ is modeled as independent complex Gaussian random variable with zero mean and unit variance.

\section{SIMULATION RESULTS}

In this section, we conduct several simulation experiments to show the performance of the proposed scheme over MIMO channels. The simulations are tested on the standard video sequence "Foreman," whose frames are in QCIF format with only luminance component. In this scheme, four descriptions are generated from the complete bitstream. For spatial decomposition, we apply the CDF 9/7 biorthogonal filter and three levels of decomposition. For overcomplete MCTF, we apply Haar filter and three levels of temporal decomposition. We adopt 3D-SPIHT core algorithm without arithmetic coding. Each tree can be encoded and decoded independently. The source coding rate is at $0.93 \mathrm{bpp}$.

We build a MIMO-OFDM simulation system as introduced in Section 2. The channel code used is a $1 / 2$ rate irregular LDPC code with a length of 6096 bits. The OFDM symbols are designed following the IEEE 802.11a standard. Specifically, each OFDM symbol occupies 64 subcarriers, in which 52 subcarriers may be used for transmitting QPSK data symbols. The frequency-selective multipath channel model is referred to as COST207 indoor model [21]. Finally, known timing and zero carrier frequency offset (CFO) are given throughout simulations. Detail simulation parameters are listed in Table 1.

\subsection{PSNR performance comparison}

In this simulation, we compare the performance of three schemes: the proposed UEP scheme, EEP scheme using only V-BLAST algorithm, and STBC scheme. PSNR performance is evaluated through several experiments. Figure 5 shows the PSNR performance of the proposed scheme compared with other schemes. In the MIMO-OFDM system without channel coding, we can achieve a great improvement. Moreover, by importing LDPC code, the SNR requirement is greatly reduced and the UEP scheme still obtains a few dB PSNR gains. It can be seen that the proposed scheme outperforms the EEP scheme. From the figure, we also observe that STBC scheme has the best performance among the three schemes. However, the transmission efficiency will drop with noticeable loss. As a result, the proposed UEP scheme can be an alternative to achieve the tradeoff between reconstructed quality and transmission efficiency for video transmission. The main advantage of the proposed scheme is that it is simpler than existing schemes using power allocation or adaptive modulation algorithm to combat with multipath fading in MIMO transmission system. Moreover, the proposed UEP scheme is more suitable for multiple antennas system than traditional UEP schemes based on channel coding.

\subsection{The performance comparison for error concealment}

We also conduct experiments to show the performance of the proposed scheme with and without error concealment over MIMO channels. Simulations are performed with channel coding and without channel coding, respectively. As discussed in the previous section, we apply the error detection strategy so as to facilitate the corresponding error concealment scheme. Error concealment scheme is only applied to the LLL frame. If we find some wavelet trees corrupted, we can compensate these "bad" wavelet coefficients by calculating the average of their surrounding wavelets coefficients in the lowest subband in the LLL frames. The other wavelet coefficients in the corrupted wavelet tree will be set to zero. Figure 6 shows the comparison of average PSNRs of 
TABLE 1: Simulation parameters.

\begin{tabular}{l|c}
\hline Antenna $(\mathrm{Tx} \times \mathrm{Rx})$ & $4 \times 4$ \\
\hline $\begin{array}{l}\text { Number of subcarriers } \\
(\text { data/all })\end{array}$ & $52 / 64$ \\
Guard interval (chips) & 16 \\
Modulation & QPSK \\
Bandwidth $(\mathrm{MHz})$ & 20 \\
Multipath latency $(\mathrm{ns})$ & $0,100,200,300,500,700$ \\
Multipath decay $(\mathrm{dB})$ & $0,-3.6,-7.2,-10.8,-18,-25.2$ \\
\hline
\end{tabular}

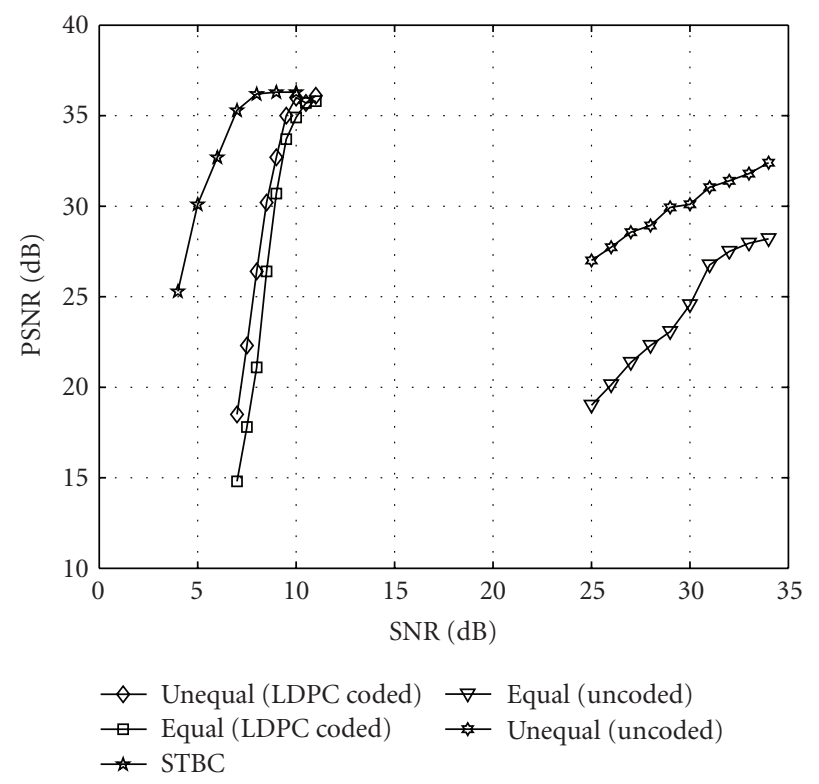

FIgURE 5: PSNR versus SNR comparison for EEP and UEP schemes.

the reconstructed frames at different SNRs with and without error concealment. Figure 7 shows both visual quality and PSNR results of some sample frames before and after error concealment. From these figures, we can demonstrate that visual quality of the reconstructed frames have been gradually improved as SNR increases, and the average PSNRs of the reconstructed frames with error concealment are higher than those without error concealment. These results show the importance of error concealment.

\section{CONCLUSION}

In this paper, we have developed a new scheme for robust video transmission over MIMO-OFDM system using MDC scheme to improve the robustness of signal source and adopting hybrid space-time coding structure to obtain unequal error protection during transmission. Experimental results have demonstrated that the proposed scheme can be an excellent alternative to achieve the tradeoff between received video quality and transmission efficiency. We believe that such an integrated approach will provide additional dimensions for optimal design of MIMO-OFDM wireless video communication systems.

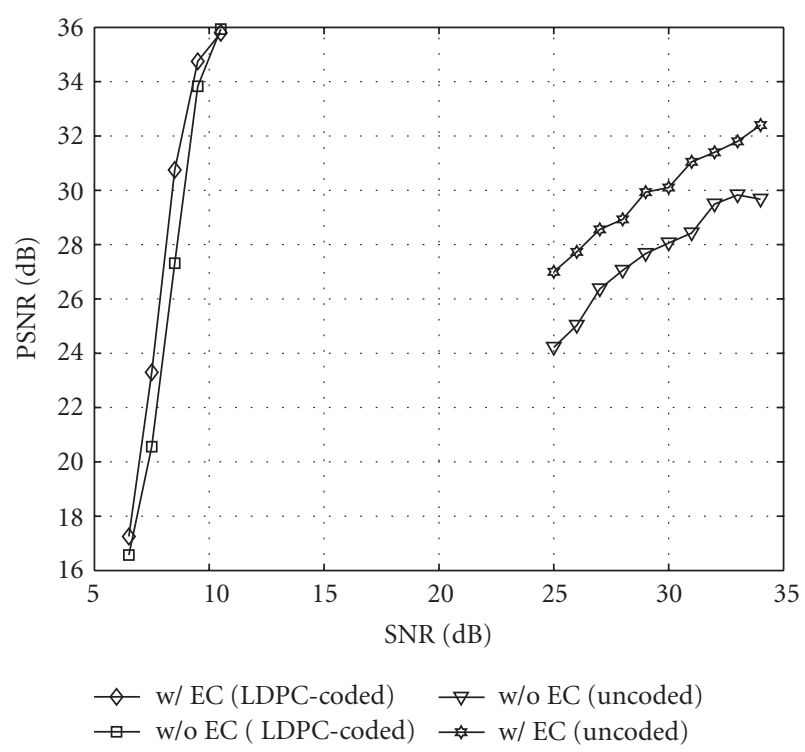

FIGURE 6: PSNR versus SNR comparison for with and without error concealment schemes.

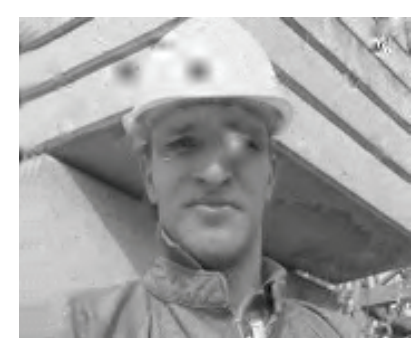

PSNR $=28.132($ before EC)

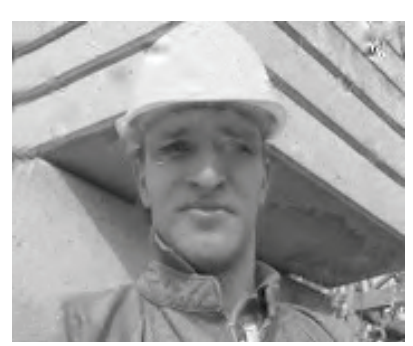

PSNR $=30.927$ (after EC) (a) $\mathrm{SNR}=33 \mathrm{~dB}$ (uncoded)

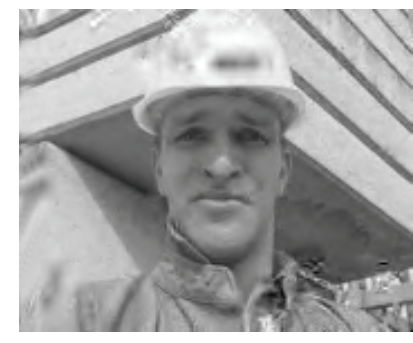

$\mathrm{PSNR}=27.433($ before $\mathrm{EC})$

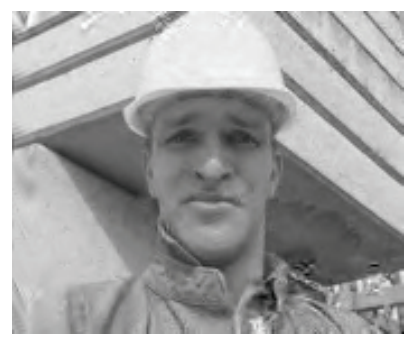

$\mathrm{PSNR}=30.855($ after EC $)$ (b) $\mathrm{SNR}=8.5 \mathrm{~dB}($ LDPC-coded $)$

FIGURE 7: Visual quality and PSNR results of some sample frames before and after error concealment.

\section{ACKNOWLEDGMENTS}

This work is supported by China National Natural Science Foundation under Research Grant no. 60328103 and the Fujian Education Department Foundation Grant no. K04004. 


\section{REFERENCES}

[1] V. Tarokh, H. Jafarkhani, and A. R. Calderbank, "Space-time block codes from orthogonal designs," IEEE Transactions on Information Theory, vol. 45, no. 5, pp. 1456-1467, 1999.

[2] G. J. Foschini, "Layered space-time architecture for wireless communication in a fading environment when using multielement antennas," Bell Labs Technical Journal, vol. 1, no. 2, pp. 41-59, 1996.

[3] H. Zheng and K. J. R. Liu, "Space-time diversity for multimedia delivery over wireless channels," in Proceedings of IEEE International Symposium on Circuits and Systems (ISCAS '00), vol. 4, pp. 285-288, Geneva, Switzerland, May 2000.

[4] H. Zheng and D. Samardzija, "Performance evaluation of indoor wireless systems using BLAST testbed," in Proceedings of IEEE Vehicular Technology Conference (VTC '01), vol. 2, pp. 905-909, Atlantic City, NJ, USA, May 2001.

[5] Z. Ji, Q. Zhang, W. Zhu, Z. Guo, and J. Lu, "Power-efficient MPEG-4 FGS video transmission over MIMO-OFDM systems," in Proceedings of IEEE International Conference on Communications (ICC '03), vol. 5, pp. 3398-3402, Anchorage, Alaska, USA, May 2003.

[6] Z. Ji, Q. Zhang, W. Zhu, J. Lu, and Y.-Q. Zhang, "Video broadcasting over MIMO-OFDM systems," in Proceedings of IEEE International Symposium on Circuits and Systems (ISCAS '03), vol. 2, pp. 844-847, Bangkok, Thailand, May 2003.

[7] C.-H. Kuo, C.-S. Kim, and C.-C. J. Kuo, "Robust video transmission over wideband wireless channel using space-time coded OFDM systems," in Proceedings of IEEE Wireless Communications and Networking Conference (WCNC '02), vol. 2, pp. 931-936, Orlando, Fla, USA, March 2002.

[8] D. W. Redmill and N. G. Kingsbury, "The EREC: an errorresilient technique for coding variable-length blocks of data," IEEE Transactions on Image Processing, vol. 5, no. 4, pp. 565574, 1996.

[9] B.-J. Kim and W. A. Pearlman, "Embedded wavelet video coder using three-dimensional set partitioning in hierarchical trees (SPIHT)," in Proceedings of Data Compression Conference (DCC '97), pp. 251-260, Snowbird, Utah, USA, March 1997.

[10] J. Liu and J. Li, "A simple soft-detector for the BLAST system," in Proceedings of IEEE Sensor Array and Multichannel Signal Processing Workshop, pp. 159-163, Rosslyn, Va, USA, August 2002.

[11] Y. Andreopoulos, A. Munteanu, J. Barbarien, M. Van Der Schaar, J. Cornelis, and P. Schelkens, "In-band motion compensated temporal filtering," Processing: Image Communication, vol. 19, no. 7, pp. 653-673, 2004, special issue on Subband/Wavelet Interframe Video Coding.

[12] N. Franchi, M. Fumagalli, R. Lancini, and S. Tubaro, "Multiple description video coding for scalable and robust transmission over IP," in Proceedings of the Packet Video Workshop (PV'03), Nantes, France, April 2003.

[13] I. V. Bajić and J. W. Woods, "Domain-based multiple description coding of images and video," in Visual Communications and Image Processing, vol. 4671 of Proceedings of SPIE, pp. 124135, San Jose, Calif, USA, January 2002.

[14] A. C. Ashwin, K. R. Ramakrishnan, and S. H. Srinivasan, "Wavelet domain residual redundancy-based descriptions," Signal Processing: Image Communication, vol. 18, no. 7, pp. 549-560, 2003.
[15] N. Tanabe and N. Farvardin, "Subband image coding using entropy-coded quantization over noisy channels," IEEE Journal of Selected Areas in Communications, vol. 10, no. 5, pp. 926943, 1992.

[16] J. M. Shapiro, "Embedded image coding using zerotrees of wavelet coefficients," IEEE Transactions on Signal Processing, vol. 41, no. 12, pp. 3445-3462, 1993.

[17] A. Said and W. A. Pearlman, "A new, fast, and efficient image codec based on set partitioning in hierarchical trees," IEEE Transactions on Circuits and Systems for Video Technology, vol. 6, no. 3, pp. 243-250, 1996.

[18] Y. Chen and W. A. Pearlman, "Three-dimensional subband coding of video using the zero-tree method," in Visual Communications and Image Processing, vol. 2727 of Proceedings of SPIE, pp. 1302-1312, Orlando, Fla, USA, March 1996.

[19] L. Cao and C. W. Chen, "Robust image transmission based on wavelet tree coding, error resilient entropy coding, and error concealment," Journal of Electronic Imaging, vol. 13, no. 3, pp. 646-653, 2004.

[20] C. Ru, L. Yin, and J. Lu, "An LDPC coded MIMO-OFDM system with simple detection and channel estimation scheme," in Proceedings of the 6th IEEE Circuits and Systems Symposium on Emerging Technologies: Frontiers of Mobile and Wireless Communication, vol. 2, pp. 693-696, Shanghai, China, May-June 2004.

[21] "Digital Land Mobile Radio Communications - COST 207," Commission of the European Communities, Final Report, Marzo 1984-Settembre 1988, Office for Official Publications of the European Communities, Luxembourg, 1989. 

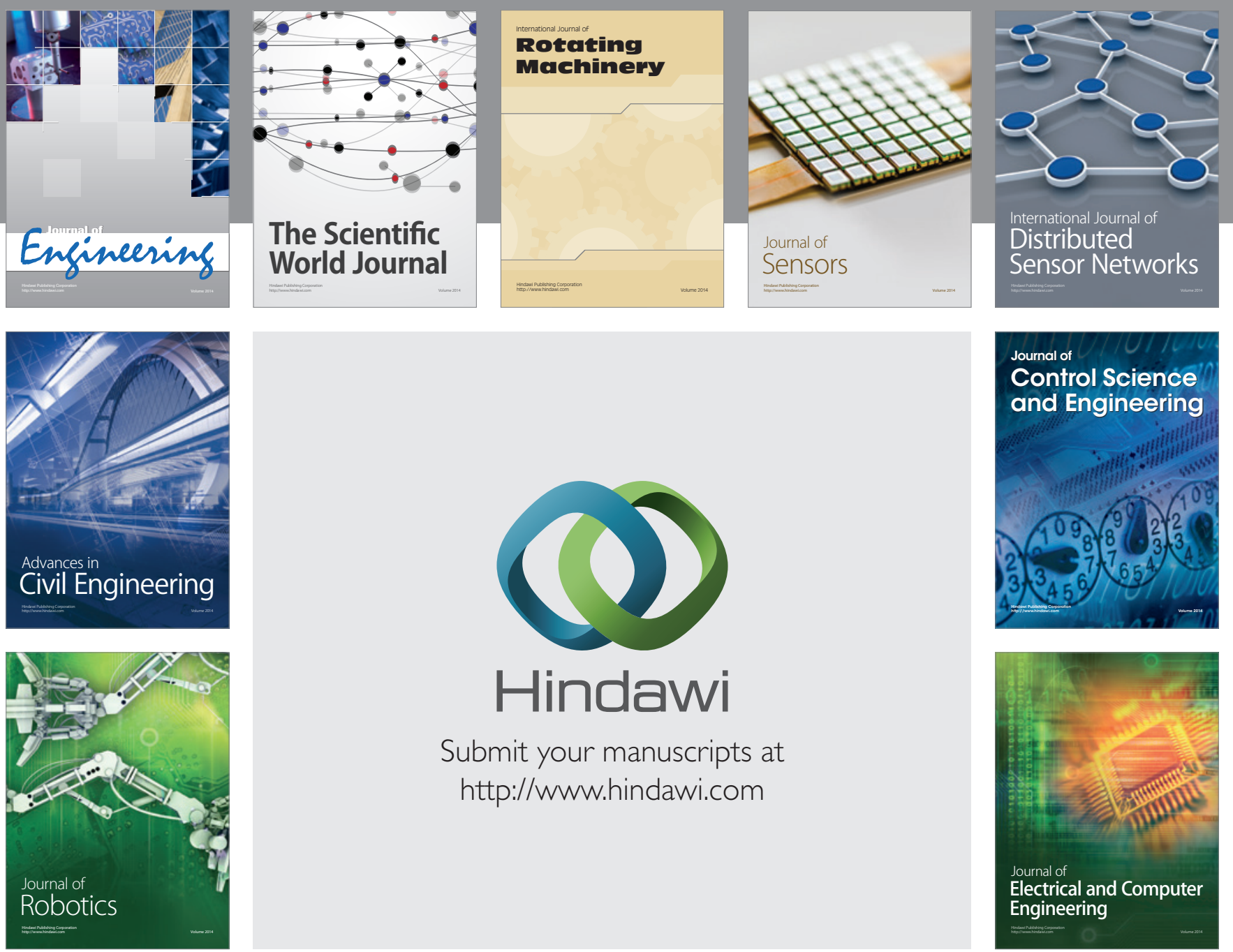

Submit your manuscripts at

http://www.hindawi.com
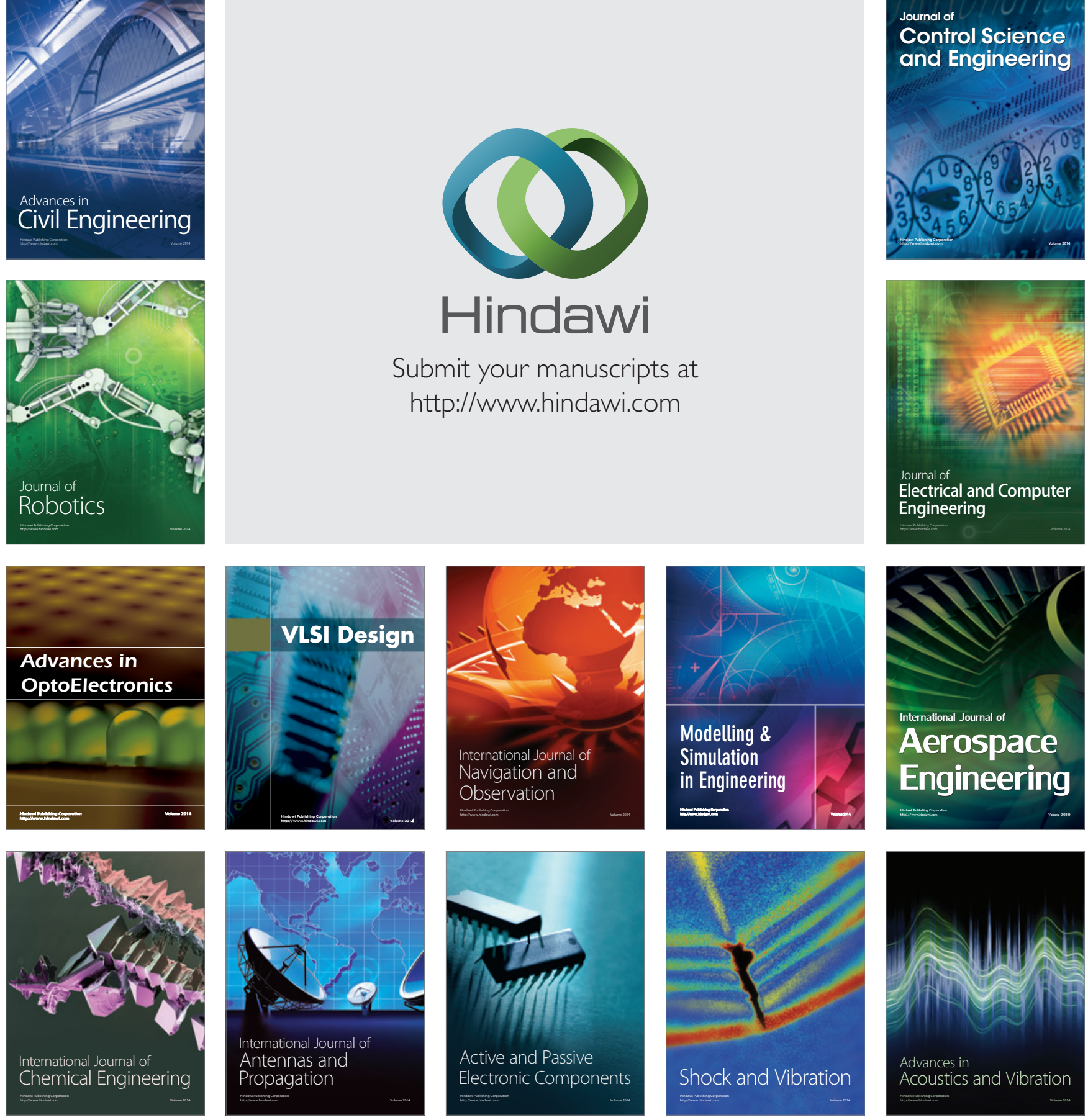